\title{
The Investment Valuation of ItsaCheckmate Company Based on PEST Analysis and POCD Method Research
}

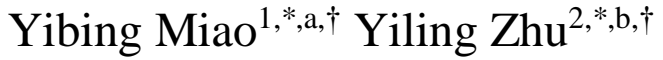 \\ ${ }^{1}$ School of Statistics and Mathematics, Central University of Finance and Economics, Beijing, China \\ ${ }^{2}$ Rutgers Business School, Rutgers University, New Brunswick, USA \\ ${ }^{*}$ Corresponding author. Email: ${ }^{a} 2017310889 @ e m a i l . c u f e . e d u . c n,{ }^{b} y z 824 @$ scarletmail.rutgers.edu \\ These authors contributed equally.
}

\begin{abstract}
As more and more food delivery platforms have met the convenience needs of online food ordering and delivery orders and are affected by the epidemic, the food ban has further stimulated the demand for sales and home delivery. ItsaCheckmate has been born. It acts as a link between the restaurant and the third-party platform. It provides an easyto-operate menu management layer for updating prices, adding, editing, or deleting items, and making menu changes across all ordering platforms from a single source. This paper uses the PEST and POCD analysis framework to assess the company's investment value. We find that based on the positive political, economic, social environment, and technology macro environment analysis, we can see the integrated food delivery platform has a sustainable development space and huge feasibility space. For itself, ItsaCheckmate has a stable team with good skills and relevant work experience. It provides cost-effective services and competes in an industry with less entrenched potential. With a good team, good service, and a promising industry, we believe that ItsaCheckmate has good investment value.
\end{abstract}

Keywords: Investment Valuation, Integrated Food Delivery Platform, PEST Analysis, POCD Framework.

\section{INTRODUCTION}

In 2020, both the Chinese and American venture capital markets reached a record high. The U.S. market's financing reached 99.48 billion U.S. dollars in 2020, with 5536 transaction cases; the Chinese market's financing scale in 2020 increased from 67.812 billion yuan in 2019 to 1.075858 billion yuan, increasing $58 \%$. Financing history records, the number of investments also increased from 3,633 to 5,019. In the $2020 \mathrm{PE} / \mathrm{VC}$ market financing round in the U.S., seed round transactions have experienced negative growth for 5 consecutive years, and the scale ratio has dropped to $25 \%$; unicorn companies have become the winners of attracting money, and in 2020 , they received a total of 44.5 billion U.S. dollars in investment, accounting for the total investment. $33.9 \%$ of the amount. In 2020, there were 184 super rounds in the U.S. with a single financing amount of more than 100 million U.S. dollars and 53 new unicorn companies with a valuation of more than 1 billion U.S. dollars. Most of the first batch of sci-tech innovation board companies have venture capital companies, and venture capital investment cases can become value mining sites.
ItsaCheckmate represents an interesting seed-stage investment opportunity. The company provides service in helping restaurants integrate 3rd party delivery platforms and make the ordering process high in efficiency and low in error rate. It integrates 3rd party delivery platforms to the existing POS systems of the restaurants, so the orders flow straight through from these 3rd party systems to the POS of the restaurant operators and print directly in the kitchen -- without any manual involvement. The company also provides an easy-tooperate menu management layer in updating prices, adding, editing, or deleting items, and synchronize menu changes across all ordering platforms from a single source. With the development, it attracts many venture capital institutes to invest. It is important to investigate the future growth of this firm.

On the study of venture capital, we found some relevant researches. GOMPERS and Paul [1] examines the structure of staged venture capital investments when agency and monitoring costs exist. Expected agency costs increase as assets become less tangible, growth options increase, and asset specificity rises. Hochberg et al. [2] find that better-networked VC firms experience significantly better fund performance, as measured by the 
proportion of successfully exited investments through an IPO or a sale to another company. Kaplan \& Strömberg [3] compare the characteristics of real-world financial contracts to their counterparts in financial contracting theory by conducting a detailed study of actual contracts between venture capitalists (VCs) and entrepreneurs. They consider VCs to be the real-world entities that most closely approximate the investors of theory. Repullo \& Suarez [4] characterizes the optimal securities for venture capital finance in an environment with multiple investment stages and double-sided moral hazard in the relationship between entrepreneurs and venture capitalists. They show that if the conditions relevant for continuation into later stages are verifiable, the optimal security gives the venture capitalist a constant share in the successful return of the project over a predetermined set of continuation states.

This article utilizes the PEST and POCD methods to study whether a company is worth investing in. There are many literatures in the existing articles about this field. Gupta [5] finds the industry life cycle is useful for explaining and predicting trends among the six forces driving industry competition. Peng \& Nunes [6] propose the use of Political, Economic, Social, and Technological (PEST) analysis as a tool to identify narrower contexts and focus research questions around feasible and meaningful regional contexts. It illustrates this process with the results of an analysis carried out as part of an ongoing Ph.D. research project. Yan \& Yi Sha [7] use the systematic analysis of the pros and cons, opportunities, and challenges from the four perspectives of PEST. The result tells us that the implementation of strengthening cooperation, increasing financial input, improving staff's qualities, using the new technology to promote cultural tourism development in Guanzhong-Tianshui economic zone. Doherty et al. [8] provide an analysis of political, economic, social, and technological factors that are impacting the Australasian higher education environment through considering the case of the Australasian Society for Computers in Learning in Tertiary Education (ascilite). Harvey Carruthers [9] discusses some of the factors that might need to be considered when applying the PEST model to veterinary practice. Barnardo et al. [10] strategically evaluate the strengths, weaknesses, threats, and resulting opportunities that face an entrepreneurial startup. To evaluate the best strategic actions to grow a business through the lens of sustainable entrepreneurship by using principles such as the triple bottom line and people, opportunity, context, and deal and framework.

In this paper, we first introduce ItsaCheckmate's background and related businesses. Then we use the PEST analysis method and POCD analysis framework to analyze ItsaCheckmate. We find that the integration takeaway platform has great room for development and feasibility of sustainable development based on the political environment, economic environment, social environment, and technology background macro environment analysis. For itself, ItsaCheckmate has a stable team with good skills and relevant work experience. It provides cost-effective services and competes in a less entrenched industry with high potential. With a good team, great service, and promising industry, we think there is a good investment value in ItsaCheckmate.

\section{FIRM DESCRIPTION}

ItsaCheckmate acts as a link between the restaurant and the third-party platform and provides an easy-tooperate menu management layer for updating prices, adding, editing, or deleting items, and making menu changes across all ordering platforms from a single source.

Since a growing number of delivery platforms have surged to cater to the needs of convenience for online ordering and takeaway orders, restaurants need to establish partnerships with as many delivery platforms as possible to not lose their customers. Besides, the ban on dine-in has further stimulated the demand for take-outs and delivering food home due to the pandemic. These factors add up to the need for a faster and more convenient ordering system for restaurants. And that is exactly what ItsaCheckmate comes for. The new idea of integrating third-party platforms has only been made possible and, more importantly, affordable for restaurants because of the development of technology and the thriving of the delivery market.

ItsaCheckmate's customers are restaurants that accept takeout orders from various online platforms. These restaurants were in a position where they need to tackle orders from the Point of Sale system, third-party delivery, and ordering platforms simultaneously. POS system is where the restaurant takes all orders in, and the delivery orders come from third-party platforms like Doordash. Other than that, multi-platform operation leads to a high error rate of order placing. If ItsaCheckmate did not exist, the restaurants would have to deal with the situation depicted in the figure. Too many tablets to work with. As a result, the cost and expenditure of the restaurant go up, but it also leads to inconvenience and low efficiency of use.

Fortunately, ItsaCheckmate provides a solution for this. On the one hand, it can be installed in more than 50 POS systems, covering almost all the popular POS systems in the market. On the other hand, it integrates over 100 ordering platforms, as DoorDash and Uber Eats, into the restaurant's POS system. ItsaCheckmate synchronizes the menus and prices directly from the POS system to the ordering platforms and integrates the orders from those platforms straight to the POS system. In this sense, it serves as a link between restaurants and thirdparty platforms. Moreover, it provides an easy-to-operate 
menu management layer in updating prices, adding, editing, or deleting items, and make menu changes across all of their ordering platforms from a single source.

As for the financial history of ItsaCheckmate, they had two rounds of financing up till now which is a Series A investment of 3 million dollars in 2018 and a Series B investment of 8 million dollars since last year (Table 1), but the funding types which raised the most money is unknown to the public. There are 2 investors in the first round of financing. The leading investor is Tiger Global Management, and the other one is the Continental Grain Company. Tiger Global mainly focuses on the internet, software, and consumer industries, exactly the one that ItsaCheckmate is in. They also have past experience in facilitating a list of successful companies like Facebook and Xiaomi. So ItsaCheckmate may benefit a lot from their experience and network. As for Continental Grain Company is a food and agribusiness investment company that understands the demand in the food supply industry due to their long history in this industry, thus bringing insights to ItsaCheckmate's business. So, from financial history's perspective, ItsaCheckmate has gained not only 3 million dollars but also the assistance it might need in its growth.

Table 1. Financing Situation of ItsaCheckmate

\begin{tabular}{lll}
\hline Financing Amount & Investors & Funding Round \\
\hline \$ 3 million & Tiger Global Management & Series A \\
& Continental Grain Company & \\
& Tiger Global Management & \\
& Continental Grain Company \\
Inspire Brands & Killion & \\
& Kitchen Fund & Series B \\
& & \\
\hline
\end{tabular}

\section{PEST ANALYSIS}

PEST is a method to analyze the external macro environment of a company from the perspective of corporate strategy from four aspects: Politics, Economics, Society, and Technology. The formulation of a company's strategy is inseparable from the macro environment. The PEST analysis method can better grasp the current situation and changing trends of the macroenvironment from all aspects, which is conducive to the use of opportunities for survival and development. The possible threats to the environment are detected early and avoided in time. For our articles, we also use pest analysis for research.

\subsection{Politics}

We mainly consider the policy support for internet development in this part. In terms of Internet and telecommunications, the Biden administration adheres to the principle of the open Internet and promises to "uniform broadband access" and "expand broadband access to every American." In his campaign platform, Biden proposed that "the United States should lead the writing of global rules for the digital age", "maintain the ability of the United States to contain cyber threats", "cooperate with other countries and the private sector to protect personal data and critical infrastructure" and "use all available Tools to deal with countries that steal US intellectual property". This shows the government's positive attitude towards supporting the development of the Internet and reflects the importance of the Internet in today's society. It has created extremely favorable development conditions for industries that require the Internet as a platform.

\subsection{Economy}

\subsubsection{The catering industry chain is mature}

The American catering industry chain has experienced more than a century of development, and the industry is relatively mature. According to statistics from the American Restaurant Association, the scale of the catering industry has grown from US $\$ 42.8$ billion in 1970 to US\$863 billion in 2019 (estimated value, accounting for $4 \%$ of US GDP), with a CAGR of $6.32 \%$; the number of employees in the catering industry is expected to reach 15.3 million people, accounting for about one-tenth of the employed population in the United States. Also, there has a high chain rate in the United States. According to the "Top 50 Catering Companies in the United States" report, the market share of the top 50 catering companies in the United States in 2016 was $27.7 \%$. Based on the 2019 operating income of the top five listed catering companies in the US, the market share of the top 5 catering companies in the United States can reach $10.9 \%$. The American restaurant industry has achieved standardized operations and efficient internal management. There are many mature catering brands, and the market is relatively concentrated.

\subsubsection{Food delivery industry develops steadily}

In the field of food delivery, according to Euromonitor International's statistics, the U.S. food delivery revenue in 2018 was 36.356 billion U.S. dollars, 
a year-on-year increase of $20.55 \%$. The CAGR from 2013 to 2018 was $12.87 \%$, accounting for $8.16 \%$ of the overall catering revenue, compared with 2013. 5.16\% increase 2.99 pct. The U.S. food delivery service market developed early and grew steadily, with a high penetration rate of food delivery (the scale of food delivery accounts for the proportion of national catering revenue).

At present, there are many third-party food delivery platforms, and the competition is fierce but at the same time geographically. There are four main participants in the food delivery business. According to data from Second Measure, in July 2019, DoorDash had a market share of $36.5 \%$, ranking first, with GrubHub having a market share of $33.3 \%$, Uber Eats having a market share of $12.8 \%$, and Postmates having a market share of $11.5 \%$. The four major platforms have their own advantageous regional divisions. For example, Grub is more popular in Northeast New York, Boston, and other places. The integrated platform can take advantage of geographical advantages to combine different take-out distribution platforms to form a platform with wider coverage, broad development prospects, and high profits.

\subsection{Society}

\subsubsection{Change in consumption concept}

With the acceleration of the Internalization process, residents' eating and consumption habits have changed, dining out and ordering for takeout are more frequent. People are pursuing health, quality, and abundance of categories. According to statistics from the American Restaurant Association in 2019, $90 \%$ of consumers like to go to restaurants for consumption, $44 \%$ of consumers order food through restaurant apps or websites in the past year, $22 \%$ prefer restaurants with internet services, and $61 \%$ prefer healthy foods, $51 \%$ prefer environmentally friendly food. With the changes in catering consumption habits, the number of users of food delivery platforms has increased rapidly, and more and more consumer areas can be reached. Catering companies have gradually realized that cooperating with professional food delivery platforms effectively increases order volume. It saves the cost of developing a food delivery system. The integrated platform is an upgraded version of the takeaway platform, allowing merchants to connect more conveniently with almost all additional selling platforms. This brings more opportunities and profits to the integration platform.

\subsubsection{The impact of the epidemic}

Due to the impact of COVID-19, many areas have issued bans, not allowing people to eat in public areas such as restaurants. This has led many people who want to eat out to choose takeout, which has promoted the development of takeout during this special period.

\subsection{Technology}

With the continuous development of the Internet and the increase in penetration rate, application sites have become more common and cover a wide range. More and more restaurants are cooperating with more than one takeaway platform to increase order volume. For restaurants, online ordering has gradually become the main source of business.

Overall, with the rapid development of the Internet today, the government attaches great importance to the development space of the Internet industry, and its policies vigorously support the Internet economy, which fully reflects the country's positive attitude towards this. According to the above macro background analysis from the political environment, economic environment, social environment, and technological environment, it can be seen that the integration of food delivery platforms has a lot of room for development and the feasibility of sustainable development. Therefore, the investment space is large, the rate of return is objective, the future development is expected, and the profit is relatively high.

\section{POCD ANALYSIS}

By going through the strengths and weaknesses of people, opportunities, and context, we believe that ItsaCheckmate presents a good investment opportunity.

\subsection{People}

From the founder and its core employees, we can see that they have a strong educational background in software engineering, marketing, and other specific areas. Also, they have various related working experience such as in Forbes Technology Council, Prayantr Technologies. In this sense, their relevant skills and working experience directly benefit ItsaCheckmate to create a good integration system and adapt to the market demand.

Also, we learned from Link-din that, over these years, employees did not change a lot. A stable team results in consistent management and company development goal. And the company could save costs for training new employees. Besides, there is no need to worry about the loss of core technology due to employee resignation, which will cause additional competitive pressure.

However, their team composition also has some weaknesses. (1) We found that there are only 11 employees in the engineering group, which is a small team compared with its competitors. More professional technical talents are needed to help the company continuously upgrade its products. (2) From various recruitment websites, we find that all positions 
ItsaCheckmate wants to fill are relevant to management, showing that they currently lack management experience. They want to fill the gap and improve the running efficiency of the business. (3) Core team members have other occupations, so they cannot fully concentrate on ItsaCheckmate. Too much work may make them confuse the tasks of different positions. Errors are more likely to occur, and their working efficiency might be low. (4) There is no data analysis department neither for the company itself nor their clients. It would then be difficult for ItsaCheckmate to understand its customers' growth rate and revenue margin. And it cannot help the restaurants to understand their business and improve their revenue either.

Firstly, they can cooperate with other tech and data analysis firms to solve these problems, and they've already partnered with Allset. In addition, they need to hire more tech talents and employees with management experience. Besides, recruiting competent assistants could help core employees streamline the work, reduce errors, and improve their working efficiency.

\subsection{Opportunity}

As for the opportunity, overall, we believe ItsaCheckmate helped to tackle the pain point of taking delivery orders, and their service is worthy of the cost based on the benefit-cost analysis. Otter estimated that if equipped with integration service, the delivery revenue for a restaurant would go up by 250 dollars a month. On the other hand, the most expensive service ItsaCheckmate provides charges 100 dollars a month, so the benefit totally outweighs the cost. Further, other costs would be incurred if the restaurant operates without the integration service.

The integration business has an edge in efficiency, etc., and lowers the cost for the restaurant. It would be a wise choice for the restaurants to subscribe to the integration service in terms of the cost. And we can expect current customers to stay, and more customers are coming in subscribing to their business in the long run.

These are the services ItsaCheckmate, and its competitors provide and corresponding relative ranking (Table 2). Here we didn't consider Chowly because their rating is too low, and customers' feedback is super negative.

Table 2. Services Ranking Comparison between ItsaCheckmate and its competitors

\begin{tabular}{lllll}
\hline & ItsaCheckmate & Otter & Ordermark & Chowly \\
\hline Mobile Menu Management & $\sqrt{ }$ & $\times$ & $\times$ & $\times$ \\
Platform Integration & 1 & 2 & 3 & $\sqrt{ }$ \\
Team Support & 1 & 3 & 2 & $\sqrt{ }$ \\
Data Analysis & $\times$ & 2 & 1 & $\times$ \\
Order Throttling & $\times$ & $\sqrt{ }$ & $\times$ & $\times$ \\
\hline
\end{tabular}

Firstly, we can see ItsaCheckmate provides menu management service on mobile phones, which would be convenient for restaurants owners to operate on. Also, it is ItsaCheckmate's exclusive service. As for the amount of platforms integration, ItsaCheckmate ranks first. Another is that ItsaCheckmate has top-tier team support. They have a dedicated customer support team available around the clock that focuses on handling everyday needs and questions of the restaurants.

However, ItsaCheckmate doesn't have a data analysis service while its competitors have. This is one of its weaknesses. However, ordinary restaurants rarely read the data and don't understand it. It doesn't matter to them if no one helps to explain the data. Currently, all these firms don't provide in-person data services. Moreover, ItsaCheckmate also doesn't facilitate order throttling while Otter does. It is a service that limiting the number of orders during the peak period. If not limiting the maximum, the order will be automatically sent to the back kitchen, which may cause disorder and burden. So this is an obvious weakness of ItsaCheckmate's service.
In terms of cost, ItsaCheckmate doesn't charge of setup fee and Otter, while Ordermark charges a lot. It seems that Otter offers a lower price with a data analysis service. But their pricing difference is marginal. It is almost less than $\$ 1$ per day. And Data analysis is only important for chain restaurants. While ItsaCheckmate excels in what it does. If they set up a great data analysis team, they would excel in the market from all aspects.

Overall, ItsaCheckmate has an obvious advantage in mobile menu management, platform integration, and team support respects. In terms of data analysis and order throttling, it still needs to be improved.

\subsection{Context}

As for the context, 2 strengths lie in the context. The delivery order market and the number of users surged in the past few years, creating a great demand for restaurant delivery orders. And restaurants have established partnerships with as many delivery platforms as possible to not lose their customers. Thus, the demand for delivery platform integration climbs as well. 
However, this is still a young industry, and the market potential has not been fully developed. Many restaurants owners still don't know about this service. If with more service promotions, such as putting more ads, some propaganda. More customers would come into service. Moreover, since the leader in the market is still in the competition, ItsaCheckmate can lower the charge for an appropriate amount in the early stages to occupy a higher market share.

\subsection{Deal}

Despite ItsaCheckmate have various pros and cons, it occupies an estimated annual revenue of $\$ 22.9 \mathrm{M}$ in the current food delivery integration market, higher than its competitors. Otter information is not available here because otter is a service project under Cloudkitchens, not even a subsidiary.

Our investment is about the current achievements and the services that can develop in the future. For example, they can hire a data analysis team to provide the report and in-person analysis. Also, order throttling should definitely be taken into consideration. Besides, to gain an edge in pricing, they can provide a discount for locations and subscription periods above a certain threshold.

Another strength of ItsaCheckmate lies in its investors. (Table 1) In series B, we are glad to see that Tiger Global and Continental Grain did follow up investment after Series A, which is a good signal, showing their confidence in the company's future. While the most conspicuous investor in this round of financing is Inspire Brands, the parent company of many chain restaurants, such as Arby's, Buffalo Wild Wings, etc. This is a distinct advantage compared with ItsaCheckmate's competitors' investors. Ordermark and Chowly are mostly invested by VC, while otter has no direct investors because it's a service provided by the firm Cloudktichens. Invested and partnered with Inspire Brands, ItsaCheckmate would have easier access to restaurants, and it doesn't have to promote its service as hard as those pure-VC-backed competitors do. Inspire Brands has already experimented with installing ItsaCheckmate in over 1000 stores of Arby's, which is a great success according to the Director of Inspire Brands and is planning to expand it to its other subsidiaries. This kind of product promotion opportunity would otherwise be hard to obtain if without investors like InspireBrands.

Overall, we recommend that we proceed with the financing. ItsaCheckmate has a stable team and is well equipped with skills and relevant working experience. It provides high value-for-money service and competes in a less entrenched industry with high potential. It is highly recommended on Reddit and has already achieved an estimate of $\$ 22.9$ million of annual revenue. This team needs more technology support and a data analyst crew. Based on all the analysis above, overall, we believe that
ItsaCheckmate has a good team, provides great services, and performs in a promising industry. We would invest in their business in the future.

\section{CONCLUSION}

This paper first introduces ItsaCheckmate's background and related business. More food distribution platforms meet the convenience needs of online food ordering and distribution orders. Due to the epidemic, the ban on food further stimulated demand for sales and door-to-door delivery. Its customers are takeaway restaurants accepting various online platforms that can be installed in over 50 POS systems, covering almost all the popular POS systems in the market. Then we use PEST analysis and POCD analysis framework to assess the company's investment value.

For PEST analysis, we divide it from four aspects: political, economic, social, and technical analysis. We have concluded that today, with the rapid development of the Internet, the government attaches great importance to the spatial development of the Internet industry. Its policy of strongly supporting the Internet economy fully reflects the positive attitude of all countries. Based on the above macro-environment analysis of the political environment, economic environment, social environment, and technical background, we can see that the integrated takeout platform has a lot of development space and the feasibility of sustainable development. So the investment space is large, the yield is objective, the future can be expected, the profit is higher.

Furthermore, considering the POCD analysis framework, it includes people, opportunities, context, and deal. We found that ItsaCheckmate has a stable team with good skills and related work experience. It provides cost-effective services and competes in a less entrained industry with high potential. We believe the company has a good team, service, and promising industry and has good investment value. In general, we think it is a company worth investing in, but there is still room for improvement.

Our research still has some deficiencies. The first shortcoming lies in the lack of quantitative models and a high proportion of statistical types. The lack of quantification makes it impossible to reasonably deduce the influence of variables, and the internal logic is not stable enough. Secondly, since the company has just gone public, the amount of publicly available data is small and difficult to obtain, which has limited reference value for research. Future research should focus on quantitative analysis and measurement models. Analyze the internal connections of different variables to facilitate investors' testing and provide a more powerful reference. 


\section{REFERENCES}

[1] GOMPERS, \& Paul, A. . (2012). Optimal investment, monitoring, and the staging of venture capital. Journal of Finance, 50(5), 1461-1489.

[2] Hochberg, Y. V., Ljungqvist, A. , \& Yang, L. U. . (2007). Whom you know matters: venture capital networks and investment performance. The Journal of Finance.

[3] Kaplan, S. N. , \& P Strömberg. Financial contracting theory meets the real world: an empirical analysis of venture capital contracts. Social Science Electronic Publishing.

[4] Repullo, R. , \& Suarez, J. . (2004). Venture capital finance: a security design approach. Review of Finance, 8(1), 75-108.

[5] Gupta, A. . (2013). Environmental and pest analysis: An approach to external business environment.

[6] Peng, G. C. , \& Nunes, M. B. . (2007). Using PEST Analysis as a Tool for Refining and Focusing Contexts for Information Systems Research. 6th European Conference on Research Methodology for Business and Management Studies.

[7] Yan, R., Yan, H., Yi-Sha, Y., \& Business, S. O. . (2014). Study on the cultural tourism development of guanzhong-tianshui economic zone_—based on the swot-pest analysis. Journal of Xi'an University of Finance and Economics.

[8] Doherty, I. , Steel, C. , \& Parrish, D. . (2012). The challenges and opportunities for professional societies in higher education in australasia: a pest analysis. Australasian Journal of Educational Technology, 28(1), 105-121.

[9] Carruthers, H. . (2009). Using pest analysis to improve business performance. In Practice.

[10] Barnardo, C. , Reyneke, M. , Ferreira, C. , \& Robertson, J. . (2021). Growbox: the reality of growth challenges for a social entrepreneur in cape town. Emerald Emerging Markets Case Studies, 11(1), 1-23. 